\title{
Las metáforas y las adivinanzas: Estudio comparativo entre dos entornos escolares ${ }^{*}$
}

\author{
Gabriela Calderón Guerrero,' Cynthia Guadalupe Maldonado Ahumada, ${ }^{2}$ \\ Martha Beatriz Soto Martínez ${ }^{3}$ \\ Universidad Autónoma de Querétaro (México)
}

Recibido: agosto 20 de 2014 - Revisado: noviembre 11 de 2014 - Aceptado: diciembre 14 de 2014

Referencia formato APA: Calderón, G., Maldonado, C. G., \& Soto, M. B. (2014). Las metáforas y las adivinanzas: Estudio comparativo entre dos entornos escolares. Revista Cientifica Guillermo de Ockham, 12(2), 51-58.

\section{Resumen}

En este artículo se explora cómo los niños comprenden las metáforas contenidas en las adivinanzas populares de la región central de México. Para ello, se seleccionaron dos escuelas (una escuela pública y una privada) de la ciudad de Querétaro, México. En cada escuela se entrevistaron treinta estudiantes (diez de segundo, diez de cuarto y diez de sexto de primaria), para un total de sesenta niños. Se les presentaron una serie de adivinanzas populares metafóricas con dos tareas: sin opciones y con opciones. Los resultados revelan que los nińos de ambas escuelas muestran una tendencia evolutiva similar en el desarrollo de la comprensión de metáforas; esto se evidencia tanto en la coordinación como en el establecimiento de relaciones conceptuales parte/todo. Además, se encontró que la modalidad de la tarea tiene un impacto poderoso en ambas poblaciones. Finalmente, si bien se observan las mismas tendencias entre ambas escuelas, los niños de la escuela con un enfoque centrado en la comprensión y producción de textos obtuvieron mejores resultados en la resolución de las adivinanzas y en la comprensión de metáforas.

Palabras clave: Metáfora, comprensión, adivinanzas, entornos educativos

\section{Metaphors and riddles: A comparative study between two school contexts}

\section{Abstract}

This research is to contrast the results obtained by children in two different school contexts (a public school and a private school) around understanding metaphors in popular riddles. Two schools (with different characteristics), from the city of Queretaro, Mexico, were selected. 30 students (10 second, 10 fourth and 10 sixth grade of primary school) were interviewed, totaling 60 . They were asked to respond to metaphorical riddles in two different tasks,

* Reporte relacionado con el proyecto de investigación El impacto del rasgo semántico disparador en la comprensión del lenguaje no literal. Registro ante el Centro de Investigación Psicológica y Educativa (CIPE): FPS201307 de la Universidad Autónoma de Querétaro. Inicio: mayo 2013. Finalización: abril de 2015.

1. Doctora en Lingüística. Profesora de la Facultad de Psicología de la Universidad Autónoma de Querétaro (México). Pertenece al cuerpo académico consolidado Psicología del Aprendizaje y Praxis Educativas y a la linea de investigación Construcción del conocimiento. E-mail: gcalderonguerrero@gmail.com

2. Licenciada Psicología y doctoranda en Aprendizaje de la Lengua y las Matemáticas. Actualmente se desempeńa como directora del preescolar México Nuevo en la ciudad de Querétaro (México). E-mail: kynthia_maldonado@hotmail.com

3. Maestra en Psicología Educativa. Es profesora de la Facultad de Psicología de la Universidad Autónoma de Querétaro (México) y coordinadora de la Biblioteca Infantil Universitaria (BIUAQ). Pertenece al cuerpo académico consolidado Psicología del Aprendizaje y Praxis Educativas y a la línea de investigación Construcción del conocimiento. E-mail: yttebsoto@yahoo.com.mx 
the first of them was spontaneous (no options) and the second one was with the support of three different response options. The results show that children of both schools show the same evolutionary tendency in the development of the understanding metaphors and increased coordination and coherence on the establishment of conceptual relations part/whole. We also found that the task mode has a powerful impact in both populations. Finally, although the same evolutionary patterns between the two schools, school children with a focus on comprehension and text production were more successful in solving riddles and metaphors.

Keywords: Metaphors, comprehension, riddles, educational environments

\section{Introducción}

La metáfora es un mecanismo cognoscitivo a través del cual se puede comprender y significar la nueva información en función de la información, saberes y conocimientos con los que ya se cuenta (Lakoff \& Johnson, 1999).

¿Es posible hablar de procesos universales que van más allá del entorno específico en el cual se desarrolle una persona? Para Lakoff y Johnson (1999), existen sistemas de metáforas universales a través de los cuales los seres humanos organizan y significan la información nueva. No obstante, también hay evidencia de que el entorno escolar tiene un efecto importante en la estructuración del léxico letrado (Nippold, 2000).

El presente trabajo se interesa por analizar las posibilidades de comprender las metáforas contenidas en adivinanzas populares por parte de nińos de primaria y conocer si las características del entorno escolar -la manera de abordar la lengua escrita en la institución - tienen algún tipo de impacto sobre la comprensión de las metáforas.

\section{La metáfora desde la perspectiva cognoscitiva}

Para la lingüística cognoscitiva, el significado es una conceptualización, una estructura semántica (Langacker, 1991) que depende de: un nivel referencial; de los dominios cognoscitivos involucrados y de la imaginería.

El nivel referencial tiene que ver con el valor convencional de las formas léxicas en cuestión y el cognoscitivo se refiere a un campo complejo de conocimiento organizado a partir del cual las formas lingüísticas son conceptualizadas (Maldonado, 1993; 2000). Este dominio configura el contexto y da forma al conjunto organizado de conocimiento y nociones asociadas, de manera más natural, a una forma o unidad léxica (Calderón, 2012). Finalmente, la imaginería es la forma como se considera la situación y genera una perspectiva (una imagen) particular, desde la que se interpretan y se vinculan los otros dos niveles del significado.
De esta forma, las metáforas constituyen operaciones cognoscitivas fundamentales a través de las cuales se conceptualiza (o se significa) la nueva información con base en información ya conocida. Ante todo, una metáfora es una relación conceptual que implica una elaboración de significado en la que se preserva totalmente una representación esquemática (altamente abstracta) que adquiere nuevos rasgos semánticos debido, principalmente, a que la expresión lingüística en cuestión está siendo aplicada en un dominio cognoscitivo con el que no se asocia naturalmente, lo que genera nuevos significados (Langacker, 1991; 2007; Maldonado, 2000).

\section{El estudio del desarrollo mental: la mirada psicológica en las relaciones parte/todo}

Para Piaget (1969), el conocimiento es resultado de la acción; es decir, de la interacción del sujeto con el objeto de conocimiento. El sujeto interpreta el mundo en función de los esquemas de asimilación con los que cuenta, pero al mismo tiempo, el mundo (el objeto de conocimiento) se resiste a ser asimilado, lo que obliga al sujeto a acomodar sus esquemas asimiladores en función de las características del objeto. De esta forma, un mismo problema puede ser analizado, conceptualizado y resuelto de forma distinta según el nivel de desarrollo de la persona. Incluso, dado el nivel de desarrollo cognoscitivo, puede haber sujetos para los que esa situación ni siquiera sea observable y mucho menos se constituya en un problema.

Es necesario entender el estado inicial del conocimiento para comprender cómo y por qué un sujeto piensa, conceptualiza y actúa frente al mundo, como lo hace en un momento determinado, tal como sucede en la interpretación de las adivinanzas de tipo metafórico. Es decir, el procesamiento mental de los adultos tiene su génesis en el procesamiento mental infantil, de ahí la importancia de estudiar su desarrollo.

El desarrollo depende de alcanzar mayores niveles de coordinación cognitiva, lo que necesariamente involucra al proceso de diferenciación e integración. Es decir,

$52 \&$ Universidad de San Buenaventura, Cali - Colombia 
todo el desarrollo cognitivo se genera en la coordinación simultánea entre esos dos aspectos opuestos y a la vez interdependientes (diferenciación e integración). Esto implica, por un lado, la paulatina diferenciación de las propiedades de las partes como necesariamente distintas al todo y distintas entre sí; y por otro lado, la paulatina integración de las propiedades de las partes entre sí y la integración de las propiedades de las partes dentro de las propiedades del todo (Piaget \& García, 1982; Ferreiro, 1997; 1999). El proceso de construcción de conocimiento supone una serie de relaciones conceptuales entre el todo y las partes, cada vez más organizadas y coherentes; es decir, un mayor nivel de integración, diferenciación y coherencia entre el todo y las partes y entre las partes entre sí, dará por resultado esquemas de conocimiento más complejos y sólidos para interactuar con el mundo.

\section{Las adivinanzas populares y la inferencia}

Las adivinanzas son juegos lingüísticos breves, presentes en una gran variedad de culturas. Son textos complejos y completos (González, 1999) en los que el adivinador debe emplear todos sus recursos (cognitivos, lingüísticos, culturales, sociales y personales) para dar una respuesta.

Diversos trabajos señalan que las adivinanzas son juegos lingüísticos que por sus características implican poner en correspondencia la información que brindan, para deducir o inferir información no explícita (Sutton-Smith, 1976; González, 1999; Calderón, Vernon \& Carrillo, 2012).

Una inferencia es una operación en la que un significado $B$ debe ser construido en función de un significado $A$ explícito (Sutton-Smith, 1976; Cain, Oakhill \& Bryant, 2004). En ese sentido, la resolución de adivinanzas requiere el establecimiento de inferencias, entre otras habilidades y competencias del adivinador, por lo que son ideales para analizar cómo se generan nuevos significados en función de la información presente.

\section{La importancia del contexto en el desarrollo}

Si bien existen aspectos inherentes al significado y a la construcción del conocimiento que impactan la comprensión de los sujetos, también es reconocido el papel que tienen los aspectos culturales específicos sobre la forma como opera la mente. La producción e interpretación de las metáforas se debe principalmente al desarrollo del léxico letrado (desarrollo del vocabulario, redes semánticas y análisis sintáctico), el cual depende estrictamente del acercamiento a textos escritos a través de agentes alfabetizadores, especialmente de la escuela y el maestro (Nippold, 2000; 2004).

Cabe señalar que desde esta perspectiva, los niños que leen con frecuencia tienen gran cercanía a los textos escritos, se interesan en una amplia gama de temas y desarrollan un vocabulario más extenso que les permite, a su vez, comprender la lengua escrita y el lenguaje no literal con más facilidad que aquellos cuyas habilidades e intereses lectores no han sido desarrollados. Así, los niños cuya educación ha promovido un contacto cercano con la lengua escrita, pueden ir más allá de los significados simples y reconocer los significados multidimensionales y simbólicos involucrados en las metáforas. En ese sentido, el papel de la escuela (y su tipo de trabajo en torno a la lengua escrita) resulta fundamental.

La escuela cumple un papel preponderante en el desarrollo lingüístico, ya que puede facilitar la adquisición de nuevo léxico que permita al niño resignificar viejos conceptos (Barriga, 2002). De esta manera, el tipo de escuela y la forma como promueve o no el contacto con la lengua escrita, se convierten en elementos que se deben considerar para el análisis de las posibilidades que tienen los niños de comprender metáforas en función del entorno educativo al que acudan.

\section{Método}

El objetivo general de este trabajo es analizar y contrastar las interpretaciones que los nińos de los grados de segundo, cuarto y sexto de primaria, de dos contextos escolares distintos, dan a las adivinanzas populares metafóricas. El propósito de este trabajo es determinar si la capacidad para comprender metáforas se desarrolla. Asimismo, interesa saber si, en caso de que se presente un patrón evolutivo, este desarrollo presenta la misma tendencia en las dos poblaciones estudiadas. Por otro lado nos interesa examinar el tipo de relaciones conceptuales y el nivel de coherencia (relaciones parte/todo) alcanzado entre las pistas por los niños para interpretar las metáforas contenidas en adivinanzas metafóricas en ambas escuelas. Finalmente, nos proponemos indagar en torno al impacto de la modalidad de la tarea (sin opciones y con opciones) y contrastar los hallazgos entre las dos poblaciones.

\section{Participantes}

Se seleccionaron dos escuelas de primaria de la ciudad de Querétaro, México. El plantel A es una escuela privada; es decir, se sostiene con las cuotas que los padres de familia 
pagan para la educación de sus hijos; el horario de clases es de $8 \mathrm{am}$ a 14:30 pm. La población que asiste a ella es de nivel socioeconómico medio y medio-alto, la escolaridad de los padres de familia es -en su mayoría- de licenciatura en adelante. En esta institución, el trabajo didáctico que se hace con la lengua escrita es intenso desde los primeros años de escuela; trabajan con un enfoque constructivista, comunicativo y funcional bajo la premisa de la construcción e interpretación de textos.

Por su parte, el plante B es una escuela pública y el horario de clases es de $8 \mathrm{am}$ a 12:30 pm. Su población es de nivel socioeconómico bajo y la escolaridad de los padres es, en promedio, de secundaria.

Se seleccionaron treinta niños por escuela: diez de segundo del platel A y diez del plantel B; diez de cuarto del plantel A y diez del plantel B; diez de sexto del plantel A y diez del plantel $\mathrm{B}$, para un total de sesenta niños.

Los niños del plantel A fueron considerados para integrar el grupo A y los del plantel B para el grupo B.

La mitad de los niños elegidos eran varones y la mitad nińas. Las edades de ambos grupos oscilaban entre los siete y los doce ańos. Todos los niños seleccionados fueron considerados por sus respectivas maestras como estudiantes promedio y se los entrevistó individualmente. La entrevista se llevó a cabo empleando el método clínico piagetiano y tuvo una duración aproximada de cuarenta y cinco minutos. Las sesiones fueron registradas manualmente y en audio para su posterior transcripción.

\section{La tarea experimental}

Dado que el propósito de la investigación es analizar si la capacidad para comprender metáforas se desarrollaba y contrastar esos resultados en función de la escuela a la que asistían los participantes, se diseńó una tarea experimental que consistía en presentar adivinanzas populares de tipo metafórico. Dichas adivinanzas no fueron diseñadas ex profeso para la investigación; por el contrario, se retomaron de la cultura popular mexicana.

La tarea se dividió en dos modalidades: una modalidad sin opciones $(\mathrm{S} / \mathrm{O})$ y una con opciones $(\mathrm{C} / \mathrm{O})$, que más adelante se detallarán.

\section{Materiales}

Si bien las adivinanzas populares son textos que vienen de la tradición oral, en esta investigación se decidió presentarlas por escrito para que los participantes pudieran retornar al texto tantas veces como fuera necesario. De esta forma, cada adivinanza fue escrita en una tarjeta (20 x $5 \mathrm{~cm}$ ) empleando letra Arial.

Para la modalidad C/O se generaron tres posibles respuestas para cada adivinanza: una de las respuestas era la esperada (metafórica), otra era un sustantivo contenido en la adivinanza y la última opción era una respuesta literal al acertijo. Cada opción fue impresa en una tarjeta $(7 \times 3 \mathrm{~cm})$, de tal manera que cada adivinanza propuesta contaba con un juego de tres opciones.

\section{Procedimiento}

Cada adivinanza debía ser leída en voz alta por el niño para brindar una respuesta espontánea (modalidad S/O). Inmediatamente después, se presentaban las tres opciones de respuesta y el niño debía confirmar o desechar su respuesta inicial, teniendo como retroalimentación las opciones que se le presentaban (modalidad C/O). El propósito de la modalidad $\mathrm{C} / \mathrm{O}$ fue analizar la actividad de los niños cuando se acotaban a tres los dominios cognoscitivos sobre los que tenían que hacer una elección -cada uno representado por una opción o respuesta tentativa- en lugar de enfrentarse a la tarea de forma totalmente libre. De esta manera, se indagó si existía o no variación frente a la respuesta inicial y a qué respondía el cambio o no de la respuesta espontánea. Cabe señalar que la aparición de un factor de apoyo (como la opción de respuesta) no siempre resulta obvia para aquellos nińos que no cuentan con las posibilidades de integrarla (Baddeley, Eisenck \& Anderson, 2010). Por lo tanto, resultaba interesante saber si los niños de los diferentes grados podían emplear las opciones a su favor y de ser así, cómo lo hacían, tanto en función del grado escolar como de la escuela de pertenencia.

Tanto en la modalidad S/O como en la modalidad $\mathrm{C} / \mathrm{O}$, se solicitaba al niño que, además de dar la respuesta que él considerara apropiada, señalara qué pista o información de la adivinanza había sido más prominente para su respuesta ("en qué te fijaste para...").

Hasta tanto el participante concluyera ambas modalidades, no se podía iniciar con el siguiente acertijo. Todas las adivinanzas fueron presentadas al azar.

\section{Resultados}

La presentación y análisis de los resultados está organizada en todos los apartados bajo la lógica de comparar lo alcanzado por los participantes de ambos contextos educativos. 
Como parte de la investigación y de acuerdo con la forma como los niños resolvían las tareas propuestas, se generó una clasificación de las respuestas de los participantes, la cual se presenta en la Tabla 1.

\section{El desarrollo de la comprensión de las metáforas en la interpretación de adivinanzas}

En función de los resultados obtenidos, es posible afirmar, en primer lugar, que efectivamente, la capacidad para comprender metáforas se desarrolla y dicho desarrollo está íntimamente ligado al grado escolar de los participantes.

En ese sentido, se observa que los niños del grupo A y los niños del grupo B se comportan de forma similar. Es decir, ambos grupos mostraron un patrón evolutivo ascendente en relación a la comprensión de las metáforas, como se observa en la Tabla 2.

Si bien la tendencia es la misma, es evidente una diferencia importante en términos porcentuales a favor de los nińos del grupo A en todos los grados. Cabe destacar que en la población del grupo $\mathrm{B}$, existe una diferencia de $33,75 \%$ entre los nińos de segundo y cuarto de primaria en las respuestas metafóricas; es decir, los alumnos de cuarto grado sextuplican la cantidad de respuestas correctas en relación con los de segundo. Por su parte, la diferencia entre segundo y cuarto (del grupo A) es solo del $17 \%$. Por otro lado, al observar a los niños de cuarto de primaria, encontramos que si bien los del grupo A obtienen mejores resultados, la diferencia respecto a los participantes del grupo B ya no resulta tan acentuada (21\%), como sucede con los participantes de segundo, cuya diferencia entre el grupo A y el B es del 37,75 \%. Esto sugiere que en el curso de tan solo dos años de escolaridad, los niños del entorno menos favorecido logran remontar de manera importante sus dificultades.

El marcado avance de los niños de cuarto del grupo B en relación con la comprensión del lenguaje no literal, puede relacionarse con el tipo de contacto que llevan a cabo los alumnos con textos escritos a partir de ese ańo escolar. En el caso del grupo A, si bien los niños más pe-

Tabla 1

Categorías de respuesta: descripción del modo de resolución

\begin{tabular}{|c|c|}
\hline Categoría & Descripción del modo de resolución \\
\hline $\begin{array}{c}1 \\
\text { (Metafórica coordinada) }\end{array}$ & $\begin{array}{l}\text { El participante logra cambio de dominio y salto metafórico al integrar e interpretar cada una de las pistas, tanto } \\
\text { literales como metafóricas de la adivinanza. }\end{array}$ \\
\hline $\begin{array}{c}2 \\
\text { (Metafórica semicoordinada) }\end{array}$ & $\begin{array}{l}\text { El nińo logra el cambio de dominio y el salto metafórico. Sin embargo, ignora alguna de las pistas o la interpreta } \\
\text { de manera incongruente; es decir, su respuesta es oportuna y bien explicada, pero puede darle una interpretación } \\
\text { literal a alguno de los rasgos que debe ser interpretado de forma no literal o viceversa; esto provoca que el partici- } \\
\text { pante rellene con información no pertinente o bien, puede ocurrir que el participante omita uno de los rasgos. }\end{array}$ \\
\hline $\begin{array}{c}3 \\
\text { (Metafórica no coordinada) }\end{array}$ & $\begin{array}{l}\text { Se ignora más de un rasgo en la adivinanza. La interpretación de las pistas parece forzada. Solo una o dos pistas } \\
\text { son la base de la respuesta de la adivinanza. Pese a lo anterior, logra el cambio de dominio y salto metafórico. }\end{array}$ \\
\hline $\begin{array}{c}4 \\
(\text { Literal })\end{array}$ & $\begin{array}{l}\text { El participante intenta dar una explicación literal y coherente a las pistas de cada uno de los versos. Sin embargo, } \\
\text { no logra el cambio de dominio y su respuesta se mantiene literal. }\end{array}$ \\
\hline $\begin{array}{c}5 \\
(\text { Literal })\end{array}$ & $\begin{array}{l}\text { La explicación del alumno es insuficiente para establecer una relación coherente entre todas las pistas. Omite } \\
\text { rasgos y otorga interpretaciones incoherentes o no integradas entre sí a las pistas. No logra el salto metafórico. }\end{array}$ \\
\hline $\begin{array}{c}6 \\
\text { (Ausencia de respuesta) }\end{array}$ & Quizá por falta de seguridad en su razonamiento o por alguna otra razón, se niega a responder a la adivinanza. \\
\hline
\end{tabular}

Tabla 2

Porcentajes de respuestas por grupo y por grado escolar

\begin{tabular}{ccccccc}
\hline & $\begin{array}{c}\text { Porcentaje de respuestas } \\
\text { metafóricas (categorías 1, 2 y 3) }\end{array}$ & $\begin{array}{c}\text { Porcentaje de respuestas literales } \\
\text { (categorías 4 y 5) }\end{array}$ & $\begin{array}{c}\text { Porcentaje sin responder } \\
\text { (categoría 6) }\end{array}$ \\
\cline { 2 - 7 } Grado escolar & Grupo A & Grupo B & Grupo A & Grupo B & Grupo A & Grupo B \\
\hline Segundo de primaria & $44 \%$ & $6,25 \%$ & $46 \%$ & $83,75 \%$ & $10 \%$ & $7,3 \%$ \\
\hline Cuarto de primaria & $61 \%$ & $40 \%$ & $31,7 \%$ & $58,75 \%$ & $1,25 \%$ \\
\hline Sexto de primaria & $82,3 \%$ & $57,5 \%$ & $12 \%$ & $37,5 \%$ & $5,6 \%$ & $5 \%$ \\
\hline
\end{tabular}


queños (segundo) muestran un nivel menos evolucionado que los de cuarto, la diferencia no es tan abrumadora como en el grupo B, lo cual se relaciona con el hecho de que la escuela a la que asisten (como ya se había señalado) trabaja constantemente y desde el inicio de la formación de sus alumnos con la lengua oral y escrita, bajo un enfoque comunicativo y de producción e interpretación de textos.

\section{Los modos de resolución de los niños: las relaciones parte/todo}

$\mathrm{Al}$ resolver un problema, cualquiera que este sea, los sujetos pueden emplear estrategias que los lleven a diferentes resultados. Aun cuando diversas estrategias o modos de resolución puedan conducir a resolver la tarea enfrentada, ese resultado puede ser más o menos logrado o sofisticado; es decir, un problema se puede resolver parcialmente o implicar una comprensión parcial (Piaget \& García, 1982). En ese sentido, habría diversos niveles o maneras de resolver algo, algunos más coherentes e integrados que otros, lo cual dependerá en buena medida, de los esquemas del sujeto. Los niveles más sofisticados de resolución de un problema, siempre implican el establecimiento de relaciones cada vez más integradoras y coherentes (Piaget \& García, 1982), lo que significa esquemas más sólidos y desarrollados. La interpretación de las adivinanzas no es la excepción. Comprender a cabalidad una adivinanza y resolverla, involucra su conceptualización como un todo, implica considerar las pistas que brinda -como sus partes constitutivas- y establecer relaciones locales entre estas (es decir, entre las pistas) y relaciones globales entre las partes y el todo (entre las pistas y la adivinanza).

Uno de los intereses de la presente investigación fue conocer cómo los niños, de acuerdo con el grado escolar (segundo, cuarto y sexto) y con el grupo (A o B), resolvían las adivinanzas y determinar si empleaban estrategias o modos de resolución distintos (Tabla 1) cuando alcanzaban respuestas metafóricas. Los datos obtenidos se muestran en la Tabla 3.
Al comparar los datos relativos a los modos de resolución encontrados en ambos grupos, podemos observar que el grupo A alcanza con mayor frecuencia las respuestas metafóricas semicoordinadas y no coordinadas. El modo de resolución tipo 3 es el más utilizado $(29,60 \%$, $33,60 \%$ y $35 \%$ por los nińos de segundo, cuarto y sexto, respectivamente) mientras que el modo de resolución tipo 1 fue el menos frecuente.

Por otro lado, el grupo B muestra el fenómeno contrario en los grados avanzados. Las respuestas menos frecuentes fueron las del modo de resolución tipo 3, a pesar de que fueron las únicas respuestas metafóricas alcanzadas por los alumnos de segundo grado con un 6,25\%. Las respuestas metafóricas más frecuentes corresponden al modo de resolución tipo 1, de las cuales el $26,25 \%$ son de los alumnos de cuarto de primaria y el $42,5 \%$ de los alumnos de sexto grado; es notoria la ausencia de ambos tipos de respuestas en los alumnos de segundo grado.

Pareciera que los participantes del grupo B, bajo ciertas condiciones, logran sacar un mayor provecho de las circunstancias que los del otro grupo. Es decir, si bien los niños más pequeños del grupo B parecen estar en desventaja frente a sus pares de la escuela $A$, los niños de los grados subsiguientes (cuarto y sexto) cuando logran interpretar las metáforas contenidas en las adivinanzas, lo hacen de una forma más sofisticada y coherente que los de la otra escuela.

\section{El impacto de la modalidad de la tarea: sin opciones y con opciones}

Es sabido que la modalidad o la forma como se presenta una tarea en situaciones de investigación psicolingüística, puede tener un impacto en la manera como los sujetos la llevan a cabo y, por lo tanto, en los resultados obtenidos (Tolchinsky, 2004; Yopp, 1988). En ese sentido, resultaba importante saber cómo la modalidad de la tarea (S/O y C/O) impactó la comprensión de la metáfora en los participantes estudiados y si los patrones que presentaban ambas poblaciones eran similares o no. Los datos se presentan en la Tabla 4.

Tabla 3

Porcentajes de los modos de resolución 1, 2 y 3 por grado y por grupo

\begin{tabular}{|c|c|c|c|c|c|c|}
\hline \multirow[b]{2}{*}{ Grado escolar } & \multicolumn{2}{|c|}{ Modo de resolución 1} & \multicolumn{2}{|c|}{ Modo de resolución 2} & \multicolumn{2}{|c|}{ Modo de resolución 3} \\
\hline & Grupo A & Grupo B & Grupo A & Grupo B & Grupo A & Grupo B \\
\hline Segundo de primaria & $3,6 \%$ & $0 \%$ & $10,6 \%$ & $0 \%$ & $29,6 \%$ & $6,25 \%$ \\
\hline Cuarto de primaria & $7,3 \%$ & $26,25 \%$ & $20 \%$ & $7,5 \%$ & $33,6 \%$ & $6,25 \%$ \\
\hline Sexto de primaria & $22,3 \%$ & $42,5 \%$ & $25 \%$ & $12,5 \%$ & $35 \%$ & $2,5 \%$ \\
\hline
\end{tabular}


Tabla 4

Porcentajes de respuestas metafóricas por grado escolar, por grupo y por modalidad de tarea

\begin{tabular}{cccc}
\hline \multirow{2}{*}{ Grado escolar } & & \multicolumn{2}{c}{ Porcentaje de respuestas metafóricas (categorías 1, 2 y 3) } \\
\cline { 2 - 4 } Segundo de primaria & Modalidad & Grupo A & Grupo B \\
\cline { 2 - 4 } & $\mathrm{S} / \mathrm{O}$ & $17,98 \%$ & $2,5 \%$ \\
\hline \multirow{2}{*}{ Cuarto de primaria } & $\mathrm{C} / \mathrm{O}$ & $68,98 \%$ & $10 \%$ \\
\cline { 2 - 4 } & $\mathrm{S} / \mathrm{O}$ & $37,98 \%$ & $22,5 \%$ \\
\hline \multirow{2}{*}{ Sexto de primaria } & $\mathrm{C} / \mathrm{O}$ & $83,97 \%$ & $57,5 \%$ \\
\cline { 2 - 4 } & $\mathrm{S} / \mathrm{O}$ & $68,55 \%$ & $35 \%$ \\
\hline
\end{tabular}

De acuerdo con la Tabla 4, el grupo A tiende a responder metafóricamente con mayor frecuencia que el grupo $\mathrm{B}$, tanto en la modalidad $\mathrm{S} / \mathrm{O}$ como en la $\mathrm{C} / \mathrm{O}$. Sin embargo, en ambas poblaciones es notorio el aumento en la cantidad de este tipo de respuestas una vez que se presentan las opciones.

Los datos antes mencionados nos permiten corroborar que la tarea tiene un fuerte impacto en los participantes y favorece, en ambos grupos, el proceso de construcción de respuestas cada vez más integradoras y coherentes, independientemente del entorno escolar.

\section{Discusión}

Uno de los debates más interesantes en relación con la cognición, el lenguaje y las operaciones que implican, gira en torno a si su origen es universal o culturalmente situado y específico. Sin ánimo de saldar esta discusión, el presente artículo arroja datos que permiten afirmar que, efectivamente, existen procesos que van más allá del entorno cultural (en este caso, el entorno escolar específico). Como se ha expuesto en la sección de resultados, encontramos que niños de escuelas con características diferentes presentan la misma tendencia en cuanto al desarrollo de la comprensión de metáforas y por ende, a la categorización y generación de los significados.

Sin embargo, los hallazgos de la presente investigación también apuntalan la importancia de las características particulares del entorno, nuevamente de la escuela. En términos más detallados, aquellos niños que cuentan con una serie de apoyos culturales (como la didáctica de la escuela centrada en la producción e interpretación de textos) también tendrán mayores posibilidades de alcanzar más tempranamente la comprensión de las metáforas, con el impacto que esto supone para la cognición en general. Sin embargo, no deja de resultar interesante que los niños de entornos menos favorecidos tienen el potencial de alcanzar a sus pares e incluso, cuando logran dar el salto metafórico, logran establecer una mayor y mejor coordinación entre las pistas semánticas (una mejor integración y coherencia entre las partes y el todo) que los niños con más apoyos. En ese sentido, esta investigación resulta esperanzadora.

De igual manera, los datos del estudio permiten afirmar que, independientemente del tipo de población de la que se trate, el hecho de acotar los dominios cognoscitivos con los que deben lidiar -en este caso, el hecho de brindarles opciones de respuesta- resulta altamente favorecedor, pues de esta forma los niños pueden centrar su actividad cognitiva a través del empleo de ciertos observables brindados por las mismas opciones y optimizar sus recursos mentales para la elección y tratamiento de la información prominente y relevante.

\section{Referencias}

Baddeley A., Eisenck, W., \& Anderson, C. (2010). Memoria. Madrid: Alianza Editorial.

Barriga, R. (2002). Estudios sobre habla infantil en los años escolares: un solecito calientote. México: El Colegio de México.

Calderón, G. (2012). La comprensión de la metáfora en niños y jóvenes: El caso de las adivinanzas. México: Universidad Autónoma de Querétaro.

Calderón, G., Vernon, S., \& Carrillo, M. (2012). Interpretación y reinterpretación de adivinanzas metafóricas: La importancia del nivel de desarrollo y del tipo de tarea en nińos de 7 a 13 ańos. Estudios de Lingüistica Aplicada, 30(56), 61-82.

Ferreiro, E. (1997). Alfabetización, teoría y práctica. México: Siglo XXI.

Ferreiro, E. (1999). Vigencia de Jean Piaget. México: Siglo XXI.

González, G. M. (1999). Hacer visible lo invisible. Estructuras y funciones de la adivinanza mexicana tradicional. México: Plaza y Valdés. 
Cain, K., Oakhill, J., \& Bryant, P. (2004). Children's reading comprehension ability: Concurrent prediction by working memory, verbal ability, and component skills. Journal of educational psychology, 96(1), 31-42.

Lakoff, G., \& Johnson, M. (1999). Metaphors we live by. Chicago: Chicago University Press.

Langacker, R. (1991). Foundations of Cognitive Grammar. Descriptive Application. Stanford: Stanford Universitiy Press.

Langacker, R. (2007). Cognitive Grammar, En D. Geeraerts \& H. Cuyckens (Ed), The Oxford Handbook of Cognitive Linguistics (pp. 421-462). New York: University Press.

Maldonado, R. (1993). La semántica en la gramática cognoscitiva. Revista Latina de Pensamiento y Lenguaje, 1(2), 157-182.

Maldonado, R. (2000). Estudios Cognoscitivos del Español. México: Universidad Autónoma de Querétaro.

Nippold, M. (2000). Language development during the adolescent years: aspects of pragmatic's, syntax and semantics. Topics in Language Disorders, 20(2), 15-28.
Nippold, M. (2004). Research on later language development: International perspectives. En R. Berman (Ed.), Language Development across Childhood and Adolescence (pp. 1-9). USA: John Benjamins Publishing Company.

Piaget, J. (1969). Biología y conocimiento. España: Siglo XXI Editores.

Piaget, J., \& García, R. (1982). Psicogénesis e historia de la ciencia. México: Siglo XXI Editores.

Sutton-Smith, B. (1976). A Developmental Structural Account of Riddles. En B. Kirshenbaltt-Gimblett (Ed.), Research and Resources for the Study of Linguistic, Creativity (pp. 111-119). Pennsylvania: University of Pennsylvania Press.

Tolchinsky, L. (2004). The nature and scope of later language development. En R. Berman (Ed.), Language Development across childhood and adolescence (pp. 233-248). USA: John Benjamins Publishing Company.

Yopp, H. (1988). The validity and reliability of phonemic awareness test. Reading Research Quarterly, 8(2), 159-177. 